\title{
Insulte et pédagogie chez les Mossi
}

Insults and upbringing among the Mossi

\section{Suzanne Lallemand}

\section{CpenEdition}

\section{Journals}

Édition électronique

URL : http://journals.openedition.org/span/192

DOI : 10.4000/span.192

ISSN : 2268-1558

\section{Éditeur}

École pratique des hautes études. Sciences humaines

\section{Édition imprimée}

Date de publication : 1 septembre 1975

Pagination : 95-104

ISSN : 0294-7080

\section{Référence électronique}

Suzanne Lallemand, "Insulte et pédagogie chez les Mossi », Systèmes de pensée en Afrique noire [En ligne], 1 | 1975, mis en ligne le 08 juillet 2013, consulté le 19 avril 2019. URL : http:// journals.openedition.org/span/192 ; DOI : 10.4000/span.192 
INSULT:- ET PEDAGOGIE CHEZ LES MOSSI

\author{
par \\ Suzanne LALLEMAND
}

FROPOS : Dans le cadre d'une étude menée depuis 1968 dans une famille mossi du Yatenga, nous avons abordé divers aspects des liens entre parents et alliés en relation avec le processus de maturation affective des enfants, et compte tenu de leur role dans l'exercice ou la surveillance du maternage et de l'éducation de ces derniers. Après avoir, en un premier temps, systématiquement utilisé le discours adulte se rapportant à ces thèmes d'enquête, nous avons ensuite choisi de recourix à l'observation directe des membres de cette communauté, que nous considérons désormais moins comme des interlocuteurs que comme des acteurs en situation. Les échanges verbaux et gestuels dont nous avons été témoins permettent une analyse qui infirme parfois le propos conscient de l'informateur sur sa pratique éducative, et révèle les spécificités de ce milieu familial sur le double plan affectif et pédagogique.

\title{
$\underline{\text { RESUME }}$
}

Un ensemble d'insultes tend à constituer une langue dans la langue dont il importe de saisir le mécanisme propre et la fonction. Une quarantaine de "situations productrices d'insultes" mettant en scène des enfants tantôt entre eux, tantôt avec leurs éducatrices, montrent que les injures d'ainé à cadet sont plus fréquentes que celles du plus jeune au plus âgé ; que la génitrice en use moins que la "mère sociale" ; cette dernière, a son tour, ne les emploie pas autant que la tante paternelle. Sur le plan formel, ces insultes se présentent rarement comme des métaphores, très fréquemment comme des métonymies auxquelles peut s'adjoindre un élément métaphorique. Sur le plan psychologique, 1'insulte apparait comme image morcelant le corps de l'enfant dont elle hypertrophie une partie, notamment la tête, le ventre et les fesses. Sur le plan sociologique, elle joue un rôle pédagogique précis (élimination des comportements prohibés) et diffus (apprentissage de la hiérarchie familiale par identification des positions respectives d'"injuriant" et d"injurié").

L'ensemble des insultes mossi peut être considéré comme un discours disposant d'un lexique (emprunté à la langue, mais relativement clos) et d'une syntaxe, ou, au moins, de procédés de construction récurrents. Il constitue donc un type d'échange 
verbal specifique - une langue dans la langue - laquelle peut a son tour se subdiviser, selon la nature de l'insulte énvisagée : celle de l'époux en colère ou de l'allié à plaisanterie diffère de celle de la femme blâmant un jeune membre du lignage, ou d'enfants se disputant entre eux. Nous $n$ 'aborderons que cette dernière "sous-langue" englobant insultes enfantines et insultes dirigées contre les enfants par les éducatrices.

A cette étude de la parole insultante doit s'adjoindre l'examen de la situation conflictuelle qu'elle dénoue, aggrave ou engendre, selon que l'on se place dans la situation de l'injuriant ou de l'injurié. Les motifs sociaux des comportements et les attitudes psychologiques qui précèdent et accompagnent ces affrontements verbaux représentent autant de contenus qu'il importe d'explorer, puis de confronter avec la forme orale qui les condense momentanément, mais ne les reflète qu'au prix. d'appauvrissementsconsidérables, et surtout, de déformations.

\section{Les acteurs des situations injurieuses.}

Ces matêriaux ont êtè collectés en 1973, dâns la cour d'une habitation regroupant les femmes qui se livrent aux activités ménagères, les fillettes qui les secondent, et les petits enfants des deux sexes, confiés à la garde des unes et des autres. Jouaient, se disputaient et travaillaient côte à côte: les derniers-nés du lignage, entre deux et trois ans ; leurs ainnées, soeurs réelles et classificatoires ; les génitrices, femmes placées sous l'autorité des "mères sociales", d'âges plus avancés et de meilleur statut familial, qui éduquent certains enfants qu'elles n'ont pas mis au monde, et interviennent dans 1 'education de tous les autres ; enfin, les femmes issues du lignage, tantes paternelles des enfants.

L'observation des menus incidents comme des querelles entre ces personnages nous ont conduit à noter une quarantaine de scènes au cours desquelles des insultes furent émises ou échangées. 


\section{Repartition des insultes.}

Entre enfants :

$$
\begin{aligned}
& \text { - de cadet à alné : } 4, \\
& \text { - d'alné à cadet : } 8 \text {. }
\end{aligned}
$$

D'enfants à adultes : 4 .

D'adultes à enfants : 25 .

- de génitrices à enfants : 4 ,

- de "mères sociales" à enfants : 8,

- de tantes paternelles à enfants : 13 .

Quelgues insultes et leurs contextes.

"Ou est Hawa ?" demande une fillette de cing.ans à une â̂née de treize ans. - "Dans tes fesses" (a be $f$ sera) répond celle-ci. "Tu es un chien" ( $f$ ya bara) dit la mère à son enfant de deux ans qui vient de défèquer sur son tapis de prière.

"Mil en touffe" (ka kidi) dit la tante paternelle à une fillette au ventre proéminent qui chahute avec une autre enfant. "Figure de boeuf" (nafo ninga) dit une "mère sociale" irritée par les pleurs d'un bébé.

Esquisse d'un traitement formel de ces insultes.

En s'inspirant des recherches sémiologiques de R. Barthes dans le système de la mode (1) l'insulte peut être l'objet d'investigations à différent's niveaux représentables par le schéma suivant :

système rhétorique

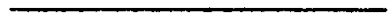

système terminologide l'injure

$\frac{\begin{array}{c}\text { signifiant } \\ \text { spect formel } \\ \text { (procédé) }\end{array}}{\begin{array}{c}\text { signifiant } \\ \text { (individu-cible) }\end{array}} \quad \begin{gathered}\begin{array}{c}\text { signifié } \\ \text { aspéologique }\end{array} \\ \text { (cẹ qui est dit de } \\ l^{\prime} \text { individu) }\end{gathered}$

Au niveau du premier système - celui que $R$. Barthes appelle aussi quelquefois celui de la langue par opposition à métalangue, celui du dénoté par rapport au connoté, nous pourrions proposer les contenus suivants : exemple : toi $=$ chien toi élément péjoratif

(1) R. BARTHES. Système de la mode. Paris, ed. du Seuil, 1967, 327 p. voir notamment le chapitre 3, "Entre les choses et les mots". 
Mais il est rare que l'élément péjoratif ne puisse se scinder à son tour ; exemple : toi = figure = figure de boeuf, toi $=$ (ventre) $=\operatorname{mil}$ en touffe.

Au niveau du second système, nous pouvons, afin de cerner les procédés de la rhétorique de l'insulte, faire appel à la distinction introduite par $R$. Jakobson entre métaphore et métonymie. Nous l'interprèterions de la façon suivante : lorsque le locuteur substitue globalement a la personne injuriée (toi) un analogue péjoratif global (chien, folle, batard), il y a métaphore ; mais lorsque l'injuriant isole, privilégie, une partie physique (figure, ventre) de l'adversaire, il procède à une métonymie. Dans notre matériel, ces deux démarches se combinent souvent sous forme de métonymie secondairement métaphorisêe ( $t u$ n'es gu'une figure, laquelle est comme celle d'un boeuf; tu n'es gu'un ventre lequel est comme une touffe de mil).

On peut aussi s'interroger sur le contenu même de ces metonymies et opposer sur ce plan I'injuriant appartenant à la catégorie des éducatrices de celui qui relève des alliés à plaisanterie. On constate que les pédagogues évoquent volontiers la tête, le ventre et les fesses de l'enfant. Mais les turbulents parents par alliance mossi (notamment le frère cadet du mari avec l'épouse de son aîné) préfèrent réduire leur partenaire à ses parties sexuelles. Le contenu métonymique de l'injure fait donc référence à une représentation spécifique du corps, dont le découpage renvoie à des zones perçues comme enfantines ou adultes, neutres ou érotiques, ainsi qu'à une idéologie des liens de parenté et d'alliance qui oriente et diversifie le vocabulaire des échanges agressifs familiaux.

\section{Aspects psychologiques de l'insulte.}

Ces métonymies utilisées par les femmes mossi dans leurs rapports avec les enfants peuvent aussi être opposées à l'insulte maternelle française : moins inventive, il semble qu'elle ait davantage recours à la métaphore (le vilain, le méchant, le bêta, l'imbécile) ; son répertoire renvoie souvent à l'être psychique, intellectuel et moral, des descendants ; inversement, les mères et les tantes mossi insistent sur le paraitre de ceux 
qu'elles eduquent. Nous sommes donc eu présence de deux éthiques, de deux conceptions de l'insulte-chatiment jont í jeconde. celle des mossi, peut etre décrite de ia façon suivante : L'acte infantile prohibé modifie (dans l'imaginaire des éducatrices, puis surtout, des enfants) le corps du jeune coupable, le morcelle d'abord, l'ampute de toutes ses parties physiques sauf une, laquelle est à son tour dégradée en objet du règne animal (figure de boeuf) ou végétal (touffe de mil). L'insulte mossi fonctionne donc comme sanction phantasmatique du geste répréhensible, en rendant immédiatement monstrueuse une partie de l'anatomie qu'elle hypertrophie et soustrait au contrôle de son propriétaire juvénile; elle punit en menaçant l'enfant de la perte de son intégrité, de son harmonie extérieure, et de sa qualité d'homme.

\section{Aspects sociologigues.}

Délaissons maintenant l'insulte pour aborder la situation dans laquelle elle vient à être formulée, et considérons les divers motifs d'affrontement des injuriants et des injuries. Un grand nombre de cas montrent ces derniers insoucieux des règles de politesse locale (enfants debout face à des gens d'âge mur assis ; enfants bruyants genant les adultes) ou rebelles aux comportements que l'on exige d'eux (ils mangent trop, ils refusent le partage). Ces conflits font apparaitre en creux, en négatif, les subtilités de l'étiquette mossi, ainsi que les normes de conduite du groupe familial.

Si l'on reprend les chiffxes relatifs aux individus habilités à manier l'injure compte tenu de leur statut familial (p.42 "répartition des insultes"), on constate que ce type de propos n'est guère récłproque, son utilisation peu égalitaire: La grande soeur injurie plus souvent un cadet que l'inverse ; l'une et I'autre, après trois ou quatre ans, ne peuvent adresser d'insultes aux adultes. De son côté, la mère a peu recours à ce type de sanction verbale; elle a à sa charge les enfants non sevrés et laisse à la "mère sociale" le soin de gronder ceux qu'elle a nourris mais n'éduque pas. Quant à la tante paternelle, son appartenance au lignage de l'enfant lui donne le 
droit de le critiquer continuellement, qu'il soit encore au sein, ou hébergé par une "mère sociale"; on ne s'étonnera pas que sorte de sa bouche le plus grand nombre d'insultes destinées aux enfants.

Aussi, si l'enfant doit, par le biais de ces sanctions verbales, progressivement distinguer les conduites permises et défendues par le groupe, il doit aussi et surtout apprendre l'organisation même de ce groupe et la position que ces partenaires de l'insulte, et lui, y occupent. Injurié face à différents types d'injuriants, injurié qui deviendra à son tour injuriant, il doit faire l'experience de la hiérarchie familiale, en intérioriser la stratification et le fonctionnement. 


\section{DISCUSSION}

L. $D E H E U S C H$ : Est-ce que les hommes adultes du lignage (grand-pēres, pères, frères) ont des injures du même type que les femmes: dans la relation femme-enfant ?

S. LALLEMAND : Ils se livrent beaucaup moins à l'injure. En fait, quand ils commencent à s'occuper de l'éducation de l'enfant celui-ci est déjà grand ; il a une dizaine d'années. I'enfant obéit et sait déja ce qu'il faut faire ou ne pas faire et, finalement, ces petites flèches injurieuses lancées contre l'enfant sont inutiles. Les relations injuriantinjurié entre père et fils sont très rares.

L. DE HEUSCH : Ce qui me frappe c'est que la société mossi semble très :: contraignante en ce qui concerne la propreté du corps. Estce très répandu dans les sociétés voisines?

S. LALLEMAND : Je n'ai pas retrouvé cette propreté chez les Kotokoli, chez qui j'ai provoqué une hilarité gênérale quand je leur ai demandé si on battait les enfants quand ils faisaient partout. On m'a répondu "Jamais, cela vient tout seul". L'adulte mosst a la réputation d'être un individu discipliné et peu joyeux, mais il faut se méfier des lieux communs.

G. $L E M O A L \quad$ : Ils sont souvent plus vrais qu'on ne l'admet.

S. $L A L L E M A N D$ : Je me souviens avoir lu dans la littérature psychologique sur I'Afrique que les Africains n'ont pas de sur-moi. Or c'est faux pour les Mossl. Ce sont des individus sévères, exigeants vis-à-vis d'eux-memes, ayant le sens de 1'étiquette.

5. DE HEUSCH : On dirait justement que ce sur-moi, pour reprendre ton schéma,ait une double réduction d'abord du corps à une partie puis de l'humanite entière à un autre domaine animal ou vegetal. Il y a comme une double régression. 
G. LE MOAL

M. CARTRY

S. LALLEMAND

G. DIETERLEN

S. LALLEMAND

G. DIETERLEN
: Est-ce que cette analyse n'est pas celle de toutes les injures de tous les systèmes du monde ? Cette réduction à une partie du tout est tout à fait classique en français. Dans la société rurale française, le vocabulaire d'injures est aussi riche que celui des Mossi, mais nous l'ignorons presque totalement, car nous sommes, si on peut dire, déculturés. Il y a aussi effectivement en français beaucoup d'injures abstraites. En fait, c'est l'élément statistique de ces injures qui serait passionnant.

: Chez les Gourmantché où il y a le même contróle très strict et très sévère des sphincters, pourtant, les plaisanteries entre alliés peuvent évoquer le "cul" : "cul sale, cul pelé..."

: Ce n'est pas un "cul" sexualisé.

: Vous n'avez pas étudié les effets bénéfiques de l'injure. Chez les Dogon, au titre de parenté à plaisanterie, l'injure est un moyen de revitalisation et d'augmentation du pouvoir de chacun. Insulter quelqu'un est le plus souvent une plaisanterie; quand il s'agit d'un enfant, cela le revigore.

: Quand un enfant a fait quelque chose de bien, on lui prend la main et on lui récite sa devise. L'effet revitalisant dont vous venez de parler est alors forcé de se produire.

: Chaque parole agit d'une façon ou d'une autre sur l'individu. La parole de l'insulte est particulièrement importante. Le jeune Gxindo de Bamba insulte joyeusement les vieux Dogon de Sanga avec lesquels il est mangu, c'est-à-dire parent à plaisanterie. Ils sont tous ravis, et plus. 1'injure est forte, meilleurs sont la réplique et l'effet. Cela fait partie d'un comportement réciproque utile.

G. LE MOAL
: Il y a deux catégories d'injures : l'injure injuriante et 1'injure rituelle. 
s. LALLEMAND: : L'Injure cathartique est reciproque, celle que je viens d'évoquer ne l'est pas. Cette vitalite, on la sent, on la voit effectivement. Quand une femme d'un grand frere se mesure avec un petit frère de son mari, il y a toute une cérémonie; la famille se rassemble. On évalue les performances verbales de chacun ; les individus se defoulent et joulssent réellement de la situation. Mais dans les injures femmes-enfants, les enfants ne jouissent nullement de la situation et ils détestent celle qui les injurie trop. Les enfants ressentent plutot un amoindrissement, on leur morcelle le corps.

D'autre part, les ascendants ne traitent jamais un enfant de batard ou de fou. Seuls les enfants entre eux ou les enfants balbutiant $v i s-a-v i s$ des ascendants peuvent le faire.

G. $L E M O A L$

S. LALLEMAND

D. JONCKERS

S. LALLEMAND

G. LE MOAL
: As-tu une idée de la part d'invention dans ces injures ?

: Certaines injures comme "fou, batard..." sont très largement usitées, mais il semble que la tante paternelle, la plus vieille femme du lignage, fasse preuve d'une créativité tout à fait réelle : "morve en natte", "morve tressée". sont de son invention.

: As-tu observé des relations particulières entre certaines génerations et les enfants, par exemple entre les grands-pères et leurs petits-enfants?

: Il y a une relation très amicale entre le grand-père et ses petits-fils, mais il semble qu'il les injurie peu ; il les agace, mais il leur parle dans une langue correcte qui ne les minimise pas.

: Il y a un code des injures. Certaines sont absolument interdites aux enfants. Que se passe-t-il si un enfant emploie une injure qui lui est interdite, par ex. : une allusion sexuelle à son grand-père. Chez les Bobo, on ne peut dire que certaines injures à son grand-père.

S. LALLEMAND : Il y a la coupure avant et après le sevrage. Avant le sevrage, tout est admis de la part d'un enfant. Après, 
beaucoup de choses lui sont interdites. La coupure est très nette.

J.F. VINCENT : Cela veut-il dire qu'un enfant non sevré peut déjà très bien s'exprimer ?

S. LALLEMAND : Non, pas vraiment. Mais son maniement d'injures est perçu comme un progrès linguistique.

J.F. VINCENT : Il est tout de même capable de manier l'injure.

L. DE HEUSCH : A quel âge est le sevrage ?

S. LALLEMAND : 3 ans.

L. DE HEUSCH : Et la propreté doit être acquise à la fin du sevrage.

S. LALLEMAND : Oui, mais on commence à éduquer I'enfant dès l'âge de 2 ans.

G. DIETERLEN : Est-ce qu'on observe un comportement spécial aux funérailles des grands-parents comme on le voit ailleurs où les enfants dansent, pilient, renversent tout sur, leur passage, ont un comportement incroyable, insultent les défunts... J'ai vu des courses de garçons complétement fardés, comme masqués, qui renversaient les calebasses, criaient, faisaient des simagrées...

D. JONCKERS : On peut même jeter le corps du grand-père.

G. LE MOAL : Dans tout le monde mandé, on vole le corps du grandpère. Il $y$ a toute une ritualisation du vol qui accompagne justement l'injure. Chez les Bobo, les vols sont gradués suivant les relations familiales.

S. LALLEMAND : Cette ritualisation du vol me rappelle quelque chose, mais il n'y a rien de semblable pour les funérailles. Il faudra que je vêrifie.

D. JONCKERS : Chez les Minyanka, les enfants peuvent injurier leurs grands-parents. Par exemple, une petite fille pourra dire à sa grand'mère avec qui elle mange:"tu manges trop ; quand vas-tu mourir ?" 